\title{
Effects of Nutritional Supplementation on Prealbumin Concentrations in Pediatric Burn Patients: A Randomized, Controlled Trial
}

\author{
Jennifer A. Williams ${ }^{1, *}$, Anna Yankina ${ }^{2}$, Yong S. Choe ${ }^{1}$, Lyudmila Dokukina ${ }^{3}$, Marina Brazol ${ }^{4}$, \\ Evgeniy Altshuler ${ }^{5}$, Mikhail Gordon ${ }^{6}$, Nikolay Ostrovskiy ${ }^{7}$, Evgeniy Kuraev ${ }^{8}$ and Barbara J. \\ Marriage $^{1}$
}

\author{
${ }^{1}$ Abbott Nutrition, Columbus, Ohio, USA \\ ${ }^{2}$ Abbott Nutrition, Moscow, Russia \\ ${ }^{3}$ Nizhniy Novgorod Research Institute of Traumatology \& Orthopedics, Nizhniy Novgorod, Russia \\ ${ }^{4}$ Children's City Hospital \#1, St. Petersburg, Russia \\ ${ }^{5}$ City Clinical Hospital \#2, Kemerovo, Russia \\ ${ }^{6}$ First Republic Clinical Hospital of Ministry of Healthcare of Udmurtia Republic, Izhevsk, Russia \\ ${ }^{7}$ City Clinical Hospital \#7, Saratov, Russia \\ ${ }^{8}$ City Hospital \#20, Rostov-na-Donu, Russia
}

\begin{abstract}
Introduction: This prospective, randomized study was designed to measure the effect of nutritional supplementation on serum prealbumin (transthyretin) in pediatric burn patients in seven burn centers in Russia. The primary measure was change in serum prealbumin from baseline to Day 7.

Methods: Fifty-four children age 1-10 years with a TBSA burn of at least 15\% were enrolled into this study. Patients had baseline serum prealbumin levels $<10 \mathrm{mg} / \mathrm{dL}$ and were capable of exclusive oral feeding within 72 hours of hospitalization. One-hundred-sixteen children were screened, with 54 meeting eligibility criteria, 28 subjects in the control group and 26 in the supplement group. Patients were randomized to receive the typical hospital diet for mild to moderate burn patients or the same diet plus additional oral nutritional supplementation. The additional enteral supplementation was 299 $\mathrm{kcal} /$ day for most of the subjects.

Results: Nutritional supplementation significantly increased serum prealbumin levels of the subjects from baseline to Day 7. At Day 7, children consuming the supplementation had a mean \pm standard error change in prealbumin concentration of $8.44 \pm 1.13 \mathrm{mg} / \mathrm{dL}$ and children on hospital diet only had a change of $5.47 \pm 0.80 \mathrm{mg} / \mathrm{dL}$, with a difference of $2.97 \mathrm{mg} / \mathrm{dL}$ $(p=0.0265)$. There were no significant differences in baseline serum prealbumin concentration, nor were there differences for adverse events or safety concerns related to study product.

Conclusions: The increase of prealbumin related to enteral supplementation may provide future guidance for nutritional management of pediatric burn patients or patients with increased energy and protein needs.
\end{abstract}

Keywords: Burns, nutrition therapy, pediatrics, prealbumin

\section{INTRODUCTION}

Each year more than 300,000 people die from fire-related burn injuries worldwide [1]. Millions more suffer from burnrelated disabilities and disfigurements with psychological, social and economic effects on both the survivors and their families. The burden of burn injury is one that falls predominately on the world's poor with $95 \%$ of fire-related deaths

${ }^{*}$ Address correspondence to this author at the Abbott Nutrition, 3300 Stelzer Road, RP3-2, Columbus, Ohio 43219; Tel: 614-624-3933;

Fax: 614-727-3933; E-mail: Jennifer.williams@abbott.com occurring in low- and middle-income countries [1]. Firerelated burns are the sixth leading cause of death among 5-14 year olds in low- and middle-income countries. More schoolaged children die of fires each year than of tuberculosis or malaria [2]. Age is a prognostic factor with a mortality rate higher in children than adults [3].

The burned body responds with an increase in stress hormones that provoke the hypermetabolic response resulting in an increase in heart rate and blood pressure, glucagon shifts from the liver, an increase in hemoglobin and plasma proteins, a decrease in coagulation, an increase in lipolysis 
and thermogenesis, and a relaxation of the smooth muscle in the gastrointestinal tract [3]. Once a burn wound exceeds 15$20 \%$ of the body surface area, a large number of systemic changes occur such as a major metabolic stress response, impaired immunity and massive fluid shifts [4], with the hypermetabolic response lasting for 9-12 months following the injury. Children are more vulnerable than adults to burn injuries because of their limited energy reserves, thinner dermis, greater body surface area, and the body's dramatic shift from growth to cellular survival [3].

One of the greatest threats to survival for burn patients is infection, and because of the relationship among nutrition, immunity and wound healing, optimal nutritional support is extremely important to these patients [4]. The provision of nutrition serves two basic functions: to provide substrate for energy production through intermediary metabolism and to provide necessary nutrients that cannot be synthesized by routine means [5]. Assessment of a burned patient's nutritional status is important but difficult due to underlying problems that can confuse the interpretation of this status, such as impaired immunity from surgery and infection; effect of wound losses and transfusions on serum proteins; changes in the rate of muscle protein breakdown; and rapid physical changes in extracellular volume that can decrease body weight [3]. Body weight is the easiest indicator of a patient's nutritional state, and the preferred biochemical marker for protein malnutrition is prealbumin [3]. Prealbu- min is considered the most sensitive indicator of nutritional status because of its rapid turnover and small total body pool, which gives a snapshot of a person's diet at a specific timepoint [6]. C-reactive protein (CRP) is a blood protein that responds to burn injury differently as a marker of inflammation during the early acute phase of burn recovery [7]. Both CRP and prealbumin have been used as prognostic indicators for identifying mortality and infection risk in patients with burn injuries [6].

This study was designed to test the effect of supplementation with a pediatric formula containing $1.5 \mathrm{kcal} / \mathrm{mL}$, in combination with a prescribed hospital diet, on outcomes for pediatric burn patients as they recovered in a hospital facility.

\section{MATERIALS AND METHODOLOGY}

\section{Subjects}

This was a randomized, controlled, dual-treatment, parallel study conducted at seven pediatric burn centers in Russia. One hundred sixteen subjects were screened from February 2010 to July 2011, and 54 of these subjects met eligibility criteria. Baseline characteristics of enrolled subjects can be found in Table 1. Enrollment was complete when the estimated sample size was met for the study. The eligibility criteria for baseline serum prealbumin less than $10 \mathrm{mg} / \mathrm{dL}$ disqualified 62 of the screened subjects who had baseline val-

Table 1. Baseline Characteristics of Subjects

\begin{tabular}{|c|c|c|c|}
\hline & Experimental Group & Control Group & Total \\
\hline \multicolumn{4}{|l|}{ Gender, n (\%) } \\
\hline Female & 14 (53.8) & $17(60.7)$ & $31(57.4)$ \\
\hline Total & $26(100.0)$ & $28(100.0)$ & $54(100.0)$ \\
\hline mean $\pm \mathrm{SEM}$ & $0.15 \pm 0.15$ & $0.25 \pm 0.19$ & \\
\hline \multicolumn{4}{|c|}{$2^{\text {nd }}$ degree burn, $\%$} \\
\hline mean \pm SEM & $9.42 \pm 1.21$ & $11.21 \pm 1.81$ & \\
\hline \multicolumn{4}{|c|}{$3^{\text {rd }}$ degree burn, $\%$} \\
\hline mean $\pm \mathrm{SEM}$ & $1.12 \pm 0.39$ & $1.25 \pm 0.35$ & \\
\hline \multicolumn{4}{|c|}{ TBSA burned \% } \\
\hline mean \pm SEM & $18.65 \pm 0.97$ & $20.39 \pm 1.41$ & \\
\hline \multicolumn{4}{|l|}{ Age (years) } \\
\hline mean \pm SEM & $2.55 \pm 0.26$ & $2.84 \pm 0.41$ & \\
\hline \multicolumn{4}{|c|}{ Body weight (kg) } \\
\hline mean \pm SEM & $13.44 \pm 0.59$ & $15.32 \pm 1.15$ & \\
\hline
\end{tabular}


ues missing or greater than or equal to $10 \mathrm{mg} / \mathrm{dL}$ (28 subjects in the control group and 26 subjects in the experimental group). Because of the relatively mild to moderate burn injuries for the patients, many of the patients were presenting at hospital admission with serum prealbumin levels in normal range (11-20 mg/dL), so the eligibility criteria was changed to only include those patients at nutritional risk, at prealbumin levels $<10 \mathrm{mg} / \mathrm{dL}$ since prealbumin was the primary outcome of the study. One subject received the wrong treatment according to the randomization schedule and their data was analyzed according to actual treatment received. The 54 intent-to-treat subjects (23 male and 31 female) had a mean age $( \pm$ SEM) of $2.70 \pm 0.25$ years and mean weight $( \pm$ SEM) of $14.42 \pm 0.67 \mathrm{~kg}$. The mean percentages ( \pm SEM) of burn injury for these subjects were the following: mean percentage of TBSA burned at $19.56 \pm 0.87$; mean percentage of $1^{\text {st }}$ degree burn at $0.20 \pm 0.12$; percentage of $2^{\text {nd }}$ degree burn at $10.35 \pm 1.10$; percentage of $3^{\text {rd }}$ degree burn at $7.81 \pm 0.88$; percentage of $4^{\text {th }}$ degree burn at $1.19 \pm 0.26$; and no subjects with $5^{\text {th }}$ degree burn. All subjects were patients admitted to the hospital within $24 \mathrm{~h}$ of burn injury, capable of oral feeding within $72 \mathrm{~h}$ of hospitalization, and had at least 15\% total body surface area (TBSA) burned with some burn depth at least $2^{\text {nd }}$ degree and no more than $5 \%$ of TBSA at $4^{\text {th }}$ degree or higher. These subjects were between 1 and $10 \mathrm{y}$ of age, had a baseline serum prealbumin concentration less than 10 $\mathrm{mg} / \mathrm{dL}$, and were expected to be hospitalized for at least 14 days. The subjects did not have a history of diabetes or stress-induced hyperglycemia, did not require ventilation or dialysis treatment, did not require parenteral feeding, were not acutely impacted or constipated, and were not taking a chronic pharmaceutical agent that affects metabolism. There were no baseline differences between the two treatment groups for gender, age, body weight, degrees of burn or percentage of TBSA burned. The study protocol was reviewed and approved by an Independent Ethics Committee, Universimed, as well as local ethics committees in Russia, and parents or legal guardians of all enrolled subjects provided informed consent before study participation. The trial was conducted in accordance with the principles of Good Clinical Practice and the Declaration of Helsinki.

\section{Study Procedures}

Study personnel at the burn centers screened potential study subjects, obtained informed consent, determined subject eligibility, and enrolled willing and eligible patients. As subjects were enrolled, they were randomized to one of two treatments via sealed, opaque envelopes containing computerized randomization sequences that were generated by the sponsor, separate from clinical study involvement. The randomization envelopes were stored in a secure location at each site. Each site had its own randomization schedule, and randomization was stratified by age (1-4 y age and 5-10 y age) and was fixed with a block size of 2 . As eligible subjects were enrolled, they were to be sequentially assigned a subject number in ascending numerical order starting with the first envelope in their appropriate age strata. The two study treatments were the typical hospital diet prescribed to pediatric burn patients as a control and the typical hospital diet plus additional liquid supplementation for oral consumption as the experimental arm. The oral supplement was designed to be consumed in addition to the hypercaloric, highprotein prescribed hospital diet. Due to the nature of the two treatments, it was impossible to implement blinding during the study, but because the primary outcome of the study was objective (serum prealbumin levels), this was not considered an issue. The liquid supplementation was vanilla in flavor and packaged in $200 \mathrm{~mL}$ plastic bottles. Each bottle contained 299 kilocalories, 8.40 grams protein, 14.94 grams fat, 32.78 grams carbohydrate, and 1.50 grams fiber (PediaSure Plus Fiber Vanilla; manufactured by Abbott Nutrition, Zwolle, Netherlands). Children ages 1-4 y in the supplementation group were to consume 1 bottle per day, and children 5-10 y were to receive 2 bottles per day during the study period. At baseline, measurements for body weight, serum prealbumin and serum CRP were obtained. After these initial measures, if a subject was in the treatment group, he or she would begin oral supplementation. For the subjects in the treatment group, the mean percent of study product consumed at Days 7 and 14 were $97.90 \pm 2.71$ and $100.05 \pm 0.43$ respectively. The hospital personnel administered and documented the intake of the study formula. Body weight, serum prealbumin and serum CRP were measured again at Day 7 and Day 14 in this fourteen-day study. The primary outcome to be measured in the study was the change in serum prealbumin from baseline to Day 7. The original primary outcome for the study was the change in serum prealbumin from baseline to Day 14; however, an interim evaluation of the data revealed a normalization of prealbumin values between Days 7 and 14 of the study because of the mild to moderate burn injuries of the patients. Secondary outcomes were change in serum prealbumin from baseline to Day 14, change in serum CRP from baseline to Day 7 and Day 14, change in body weight during the study, and the number of wound infections during the study. All serum samples were analyzed by INVITRO Independent Laboratory (Moscow, Russia) with immunoturbidimetric methodology (Architect ci8200).

\section{Statistics}

There were two interim evaluations of the data performed during the study, one was unplanned and the other was planned. Because the study was unblinded, the data was reviewed to determine the suitability of subject eligibility criteria and study outcomes. Based on the summary statistics of interim evaluation data, a study sample size of 50 subjects with non-missing values for the primary variable was determined. A total sample size of 50 (25 per treatment group) would have $80 \%$ power to detect a difference in means of $3.64 \mathrm{mg} / \mathrm{dL}$ (for serum prealbumin) assuming the common standard deviation is $4.43 \mathrm{mg} / \mathrm{dL}$ using a two group t-test with a 0.05 two-sided significance level (nQuery Advisor ${ }^{\circledR}$ 5.0). The continuous variables were analyzed using analysis of variance (and covariance), repeated measures analysis of covariance, and Wilcoxon's rank sum test. The categorical variables were analyzed using Cochran-Mantel-Haenszel test and Fisher's exact test. Hypothesis tests for treatment effects 
and interaction were conducted using two-sided, 0.05 and 0.10 level tests respectively. Statistical software SAS release 9.1.3 (SAS Institute Inc, Cary, NC) was used for the analyses.

\section{RESULTS}

There were 26 subjects in the supplementation group and 28 subjects in the hospital diet (control) group. No significant differences were observed between the two groups for baseline serum prealbumin concentrations, with mean \pm SEM values of $7.84 \pm 0.26 \mathrm{mg} / \mathrm{dL}$ and $7.60 \pm 0.33 \mathrm{mg} / \mathrm{dL}$ for the control and experimental groups respectively. All blood data is presented in Table $\mathbf{2}$, along with the calculated changes in prealbumin and CRP from baseline over the study period. The difference between treatments for the primary outcome, change in serum prealbumin from baseline to Day
7, is presented in Fig. (1). The difference was statistically significant $(\mathrm{p}=0.0265)$ and the mean changes were $8.44 \pm$ $1.13 \mathrm{mg} / \mathrm{dL}$ for the experimental group and $5.47 \pm 0.80$ $\mathrm{mg} / \mathrm{dL}$ for the control group, with a difference between the two groups of $2.97 \mathrm{mg} / \mathrm{dL}$ over the initial 7 day period. There were no significant differences between the two treatments for change in serum prealbumin from baseline to Day 14 , for change in serum CRP from baseline to Day 7 or baseline to Day 14, change in body weight during the study, or number of wound infections. While there appear to be differences between the treatment groups for baseline CRP and change from baseline CRP values, the differences were not statistically significant. Although not statistically significant, there was a mean decrease in body weight from baseline through Day 14 for the hospital diet group, and a mean increase in body weight for the experimental group. Supplementation resulted in a mean increase in body weight of 148

Table 2. Blood serum data for subjects from baseline to Day 14

\begin{tabular}{|l|c|c|}
\hline \multicolumn{1}{|c|}{ Analyte } & Hospital Diet & Hospital Diet + Supplementation \\
\hline \hline Baseline Serum prealbumin, $\mathbf{~ m g / d L ~}$ & $7.84 \pm 0.26$ & $7.60 \pm 0.33$ \\
\hline Serum prealbumin at Day 7, $\mathbf{~ m g / d L ~}$ & $13.23 \pm 0.83$ & $16.22 \pm 1.07$ \\
\hline$\Delta$ from Baseline to Day 7 & $5.47 \pm 0.80$ & $8.44 \pm 1.13^{*}$ \\
\hline Serum prealbumin at Day 14, $\mathbf{~ g / d L ~}$ & $18.36 \pm 0.82$ & $18.45 \pm 0.94$ \\
\hline$\Delta$ from Baseline to Day 14 & $10.60 \pm 0.89$ & $10.65 \pm 1.03$ \\
\hline Baseline Serum C-reactive protein, $\mathbf{~ m g / L ~}$ & $47.81 \pm 6.81$ & $75.72 \pm 15.87$ \\
\hline Serum C-reactive protein at Day 7, $\mathbf{~ m g / L ~}$ & $17.40 \pm 4.00$ & $19.34 \pm 4.63$ \\
\hline$\Delta$ from Baseline to Day 7 & $-31.56 \pm 7.49$ & $-57.34 \pm 15.16$ \\
\hline Serum C-reactive protein at Day 14, $\mathbf{~ m g / L ~}$ & $3.40 \pm 0.88$ & $3.43 \pm 1.14$ \\
\hline$\Delta$ from Baseline to Day $\mathbf{1 4}$ & $-46.15 \pm 7.38$ & $-73.80 \pm 16.93$ \\
\hline
\end{tabular}

Values are mean \pm SEM

* denotes a significant difference between treatments, $\mathrm{p}=0.0265$

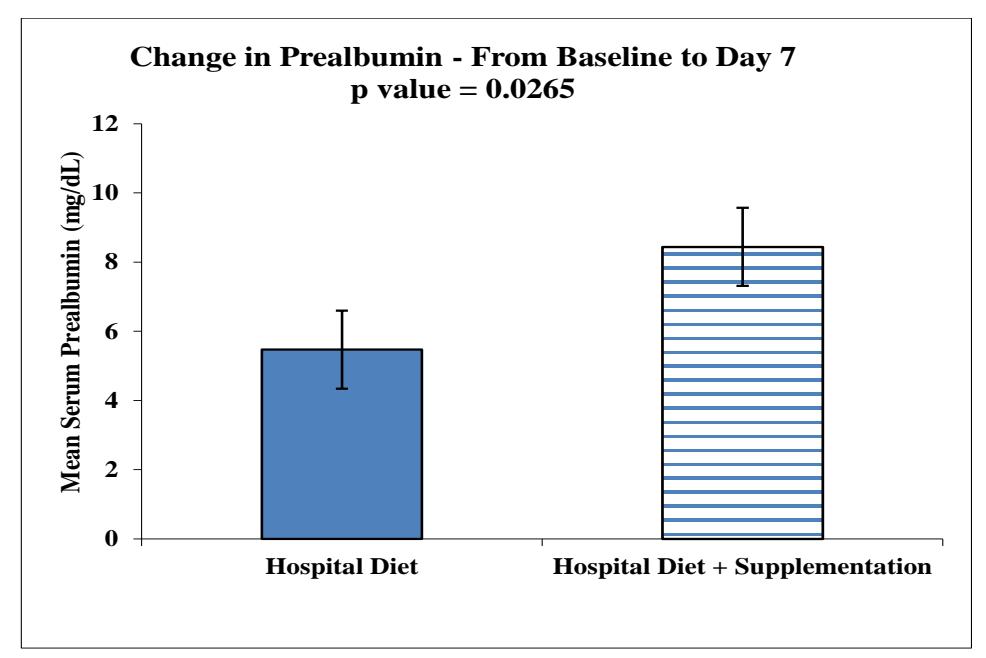

Fig. (1). Comparison of two treatments for change in serum prealbumin from baseline to Day 7 (least squares mean \pm SEM values were 5.47 $\pm 0.80 \mathrm{mg} / \mathrm{dL}$ for the hospital diet and $8.44 \pm 1.13 \mathrm{mg} / \mathrm{dL}$ for hospital diet with supplementation). 
g at Day 7 and 179 g at Day 14 compared with a mean decrease of $57 \mathrm{~g}$ at Day 7 and $56 \mathrm{~g}$ at Day 14 in the hospital diet group. There was only one subject having a wound infection during the study, which was in the hospital diet group. There were no statistically significant differences between the two groups for recorded adverse events or serious adverse events during the study. Most adverse events were considered unrelated to study product, and no concerns were noted for safety of the study product.

\section{DISCUSSION}

The current recommendation for the treatment of burn injuries is early enteral nutrition (EN). The American Burn Association advocates EN as soon as practical [8]. The Canadian Clinical Practice Guidelines recommend starting EN within 24 to 48 hours after admission to the intensive care unit in critically ill patients [9]. Early enteral nutritional support in severe burns has demonstrated numerous advantages, such as increased caloric intake, insulin secretion and protein retention, improved bowel mucosal integrity, and decreased incidence of stress gastritis [10].

Because the registration of the commercial study product in Russia required that the nutritional supplement be used and consumed in the clinical study as marketed - as an oral feed, the subjects did not have severe burns. As a result, the subjects had mild to moderate burn injuries, and the effects of supplementation on outcomes in children with more severe burns are unknown, a limitation to the results herein. Another limitation with regards to the severity of burns is the lack of difference between the two treatments for Day 14 prealbumin levels. If study subjects had more moderate to severe burns, greater or more prolonged differences in prealbumin levels may have been observed. With regards to early EN via oral supplementation, the study protocol required that subjects be hospitalized within 24 hours of burn injury and were able to orally feed within 72 hours of hospitalization. All but six subjects (11\% of the total sample) started EN within 24-48 hours of burn injury. These six subjects were admitted to regional clinics with burn injury and then transferred to a centralized burn center, and began EN within 72 hours of admittance to regional clinic. As a result, this study was a good example of the positive effects that can be attributed to early enteral nutrition via oral feeding in pediatric burn patients.

Because of the close relationship mentioned previously between nutritional status, often measured by serum prealbumin, and the risk of mortality or infection in burn patients, a positive change in this status is noteworthy, especially in the initial 7-10 days of recovery. While the amount of calories provided to study subjects in the treatment group on a daily basis was not large in quantity (299 kcal/day for 24 patients and $598 \mathrm{kcal} /$ day for 2 older patients), a clinical and statistical difference was observed with this supplementation. This supplementation was in addition to a typical hospital diet that was designed for the recovery of a burn patient. This prescribed hospital diet differed greatly in amounts of energy and protein provided, among the seven Russian hospitals used in the study. Unfortunately, because there were no dietitians or support staff in the Russian burn hospitals that were able to assist with the collection for the nutritional intakes of study subjects, the amount of hospital diet consumed is unknown for both groups. According to current ASPEN Clinical Guidelines, the quantities of protein recommended for critically ill neonates and children are based on limited data, but certain severely stressed states such as significant burn injury may require additional protein supplementation to meet metabolic demands [11]. One commonly used formula for the nutritional management of pediatric burn patients, the Curreri junior formula, calculates the caloric requirements of children age 1-3 years as basal metabolic rate (BMR) + 25 kilocalories/\% of burn and for children age 4-15 years as BMR +40 kilocalories/\% of burn [12].

\section{CONCLUSION}

While the amount of energy and protein consumed via hospital diet is unknown for the two treatment groups, the additional nutritional supplementation appears to have a positive effect on recovery from mild to moderate burn injury, whether this is due to a replacement of the hospital diet with the supplementation or because of additional nutrition in combination with the hospital diet. This study shows evidence for an enhanced solution to providing nutritional support to patients in a compromised metabolic state.

\section{CONFLICTS OF INTEREST}

Contributors: JAW designed the study, analyzed the data, and drafted and revised the paper. She is guarantor. AY helped design the study, monitored data collection for the whole trial, and reviewed the paper. YSC wrote the statistical analysis plan, analyzed the data, and reviewed the paper. LD, MB, EA, MG, NO, and EK helped with the design of the study and implemented the trial in Russia. BJM helped design the study and revised the draft paper.

\section{ACKNOWLEDGEMENTS}

Authors JAW, AY, YSC and BJM are employees of the sponsor Abbott Nutrition. Abbott Nutrition provided financial sponsorship and oversight for the trial.

\section{REFERENCES}

[1] Peck M, Molnar J, Swart D. A global plan for burn prevention and care. Bull World Health Org 2009; 87.

[2] World Health Organization. A WHO plan for burn prevention and care. Geneva, Switzerland 2008.

[3] Chan MM, Chan GM. Nutritional therapy for burns in children and adults. Nutrition 2009; 25: 261-9.

[4] Deitch EA. Nutritional support of the burn patient. Nutr Crit Ill Pat 1995; 11: 735-50.

[5] Schulman CI, Ivascu FA. Nutritional and metabolic consequences in the pediatric burn patient. J Cran Surg 2008; 19: 891-4.

[6] Gottschlich MM, Baumer T, Jenkins M, Khoury J, Warden GD. The prognostic value of nutritional and inflammatory indices in patients with burns. J Burn Care Rehab 1992; 13: 105-13.

[7] Prelack K, Dylewski M, Sheridan RL. Practical guidelines for nutritional management of burn injury and recovery. Burns 2007; 33: 14-24.

[8] Evidence-Based Guidelines Group ABA. Practice guidelines for burn care. J Burn Care Rehabil 2001; 22: 59S-66S. 
[9] Heyland DK, Dhaliwal R, Drover JW, et al. Canadian clinical practice guidelines for nutrition support in mechanically ventilated, critically ill adult patients. J Parenter Enteral Nutr 2003; 27: 35573.

[10] Mosier MJ, Pham TN, Klein MB, et al. Early enteral nutrition in burns: Compliance with guidelines and associated outcomes in a multicenter study. J Burn Care Res 2011; 32: 104-9.
[11] Mehta NM, Compher C, ASPEN Board of Directors. A.S.P.E.N. Clinical Guidelines: Nutrition support of the critically ill child. J Parenter Enteral Nutr 2009; 33: 260-276.

[12] Sharma RK, Parashar A. Special consideration in paediatric burn patients. Indian J Plast Surg 2010; 43: S43-50.

(C) Williams et al.; Licensee Bentham Open

This is an open access article licensed under the terms of the Creative Commons Attribution Non-Commercial License (http://creativecommons.org/licenses/by-nc/3.0/) which permits unrestricted, non-commercial use, distribution and reproduction in any medium, provided the work is properly cited. 